Review

\title{
Mechanisms of action for the anti-obesogenic activities of phytochemicals
}

\author{
Bilal Ahmad ${ }^{\mathrm{a}}$, Emily P. Friar ${ }^{\mathrm{b}}$, Muhammad Sufyan Vohra ${ }^{\mathrm{e}}$, Michelle D. Garrett ${ }^{\mathrm{c}}$, \\ Christopher J. Serpell ${ }^{\mathrm{b}}$, Isabel Lim Fong ${ }^{\mathrm{d}}$, Eng Hwa Wong e," \\ ${ }^{a}$ School of Biosciences, Faculty of Health and Medical Sciences Taylor's University Lakeside Campus, No1 Jalan Taylor's, 47500, Subang Jaya, Malaysia \\ ${ }^{\mathrm{b}}$ School of Physical Sciences, Ingram Building, University of Kent, Canterbury, Kent, CT2 7NH, United Kingdom \\ ${ }^{\mathrm{c}}$ School of Biosciences, Stacey Building, University of Kent, Canterbury, Kent, CT2 7NJ, United Kingdom \\ d Department of Paraclinical Sciences, Faculty of Medicine and Health Sciences, Universiti Malaysia Sarawak (UNIMAS), 94300, Kota Samarahan, Sarawak, Malaysia \\ ${ }^{\mathrm{e}}$ School of Medicine, Faculty of Health and Medical Sciences Taylor's University Lakeside Campus, No 1 Jalan Taylor's, 47500, Subang Jaya, Malaysia
}

\section{A R T I C L E I N F O}

\section{Keywords:}

Anti-obesogenic

Phytochemicals

Pancreatic lipase

Adipose tissue

Leptin/ghrelin

\begin{abstract}
A B S T R A C T
The prevalence of obesity is increasing rapidly globally and has recently reached pandemic proportions. It is a multifactorial disorder linked to a number of non-communicable diseases such as type-2 diabetes, cardiovascular disease, and cancer. Over-nutrition and a sedentary lifestyle are considered the most significant causes of obesity; a healthy lifestyle and behavioural interventions are the most powerful ways to achieve successful weight loss, but to maintain this in the long term can prove difficult for many individuals, without medical intervention. Various pharmacological anti-obesogenic drugs have been tested and marketed in the past and have been moderately successful in the management of obesity, but their adverse effects on human health often outweigh the benefits. Natural products from plants, either in the form of crude extracts or purified phytochemicals, have been shown to have anti-obesogenic properties and are generally considered as nontoxic and cost-effective compared to synthetic alternatives. These plant products combat obesity by targeting the various pathways and/or regulatory functions intricately linked to obesity. Their mechanisms of action include inhibition of pancreatic lipase activities, an increase in energy expenditure, appetite regulation, lipolytic effects, and inhibition of white adipose tissue development. In this review, we discuss the distinct anti-obesogenic properties of recently reported plant extracts and specific bioactive compounds, along with their molecular mechanisms of action. This review will provide a common platform for understanding the different causes of obesity and the possible approaches to using plant products in tackling this worldwide health issue.
\end{abstract}

\section{Introduction}

Obesity can be defined as a body mass index (BMI; weight in kg/ square of the body height in metres) of over $30 \mathrm{~kg} / \mathrm{m}^{2}$ (Khalilpourfarshbafi et al., 2018). Obesity is an international issue and a risk factor for other diseases including cardiovascular diseases, cancer, type-2 diabetes, asthma, atherosclerosis, and non-alcoholic fatty liver disease (NAFLD) (Corrêa and Rogero, 2019; Peters et al., 2018; Unamuno et al., 2018). Obesity and being overweight are the fifth leading causes of death globally (Chandrasekaran et al., 2012). According to one estimation, approximately 921 million people were obese or overweight throughout the world in 1980 and by 2013 this number had increased to 2.1 billion (Castillo et al., 2019; Ng et al., 2014) increasing from approximately $20 \%-30 \%$ of the global population. It is now widely acknowledged that the imbalance between the intake and expenditure of energy results in obesity (Ahmad et al., 2020; Parray et al., 2018).

Sedentary lifestyles and other environmental risk factors are considered major causes of obesity (Fig. 1) but they are not entirely responsible: genetic mutations can also lead to the condition (Choquet and Meyre, 2011). Mutations in genes such as Leptin (LEP), leptin receptor (LEPR), prohormone convertase 1 (PCSK1), melanocortin receptor (MC4R), proopiomelanocortin (POMC), single-minded homolog 1 (SIM), and brain-derived neurotrophic factor (BDNF) have been reported to be associated with obesity (Choquet and Meyre, 2011; Farooqi and O'rahilly, 2008).

There are a number of options for the treatment of obesity. These focus on changes in lifestyle, such as control of diet, physical exercise, weight-loss medications, and weight-loss surgeries (Chandrasekaran et al., 2012; Sun et al., 2016). Physiological interventions such as exercise and dieting are the preferred ways to reverse weight gain, but due

\footnotetext{
* Corresponding author.

E-mail address: EngHwa.Wong@taylors.edu.my (E.H. Wong).
} 